\title{
Arms Down Cone Beam CT Hepatic Angiography: Are We Focusing on the Wrong Target?
}

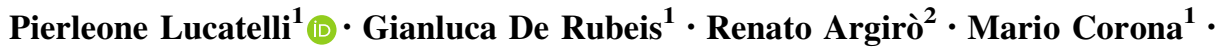 \\ Mario Bezzi ${ }^{1}$
}

Received: 21 February 2018/ Accepted: 28 March 2018/Published online: 12 April 2018

(C) Springer Science+Business Media, LLC, part of Springer Nature and the Cardiovascular and Interventional Radiological Society of Europe (CIRSE) 2018

\section{Dear Editor,}

We read with great interest the recent article by Dr. Gonzalez-Aguirre and colleagues entitled "Arms Down Cone Beam CT Hepatic Angiography Performance Assessment: Vascular Imaging Quality and Imaging Artefacts" [1]. One of the most important advantages of cone beam CT (CBCT) is the possibility to evaluate the lesion's feeders assisting their identification and catheterization [2]. In this set, the patient's arms positioning is crucial in order not to impair CBCT imaging. Dr. Gonzalez-Aguirre et al. had elegantly demonstrated that vessels' visualization is independent from the patient's arms position, allowing to perform the entire procedure without patient's movements. This minimizes the risk of contamination and reduces procedural time.

Pierleone Lucatelli

Pierleone.lucatelli@gmail.com

Gianluca De Rubeis

Derubeis.gianluca@gmail.com

Renato Argirò

Renato.argiro@gmail.com

Mario Corona

Mario.corona@uniroma1.it

Mario Bezzi

Mario.bezzi@gmail.com

1 Vascular and Interventional Radiology Unit, Department of Radiological, Oncological and Anatomo-Pathological Sciences, "Sapienza" University of Rome, Viale del Policlinico, 155, 00161 Rome, Italy

2 Department of Diagnostic and Interventional Radiology, University Campus Bio-Medico of Rome, Via Álvaro del Portillo, 21, Rome, Italy
However, literature shows that the major pivotal strength of CBCT, either mono-phasic or possibly bi-phasic, is the ability to depict in intra-procedurally "occult lesions", not visible at pre-procedural second-line non-invasive imaging (MRI, MDCT) [3]. This ability is not just for show, but yield to some major clinical implications: the visualization of an occult nodule identifies a subset of population experiencing fast tumour growth, having consequences on the number of adjunctive treatments controlling tumour growth (adjunctive RFA, or TACE procedures) and prioritization for transplantation [4]. Moreover, bi-phasic CBCT, with its unique ability to intra-procedural permit nodule characterization, could help in patients' reclassification and real-time TACE strategy modification [5].

In this light would be a crucial interest for the audience to know whether the CBCT acquisition with arms down does not alter the diagnostic performance of the modality and ability of lesion's characterization, especially for those lesion localized peripherally, where the beam hardening artefacts have been shown to be significant.

Finally, patient's positioning is fundamental for CBCT imaging. By acquiring the scan with patient's arm down, liver volume would not be located within the rotation isocentre. This could be a substantial limitation for lesion located within the left liver lobe, eventually hypertrophied, and for high BMI patients.

\section{Compliance with Ethical Standards}

Conflict of interest All authors declare that they have no conflict of interest.

Ethical Approval Statement All procedures performed in studies involving human participants were in accordance with the ethical standards of the institutional and/or national research committee and 
with the 1964 Declaration of Helsinki and its later amendments or comparable ethical standards.

Informed Consent Statement The local institutional review board approved this study. All patients underwent full informed consent for both chemoembolization procedures and study enrolment.

\section{References}

1. Gonzalez-Aguirre AJ, Petre EN, Hsu M, Moskowitz CS, Solomon SB, Durack JC. Arms down cone beam CT hepatic angiography performance assessment: vascular imaging quality and imaging artifacts. Cardiovasc Interv Radiol. 2018. https://doi.org/10.1007/ s00270-017-1875-y.
2. Cornelis FH, Borgheresi A, Petre EN, et al. Hepatic arterial embolization using cone beam CT with tumor feeding vessel detection software: impact on hepatocellular carcinoma response. Cardiovasc Interv Radiol. 2018;41(1):104-11.

3. Lucatelli P, Argiro R, Ginanni Corradini S, et al. Comparison of image quality and diagnostic performance of cone-beam CT during drug-eluting embolic transarterial chemoembolization and multidetector CT in the detection of hepatocellular carcinoma. J Vasc Interv Radiol JVIR. 2017;28(7):978-86.

4. Lucatelli P, Corona M, Argiro R, et al. Impact of 3D rotational angiography on liver embolization procedures: review of technique and applications. Cardiovasc Interv Radiol. 2015;38(3):523-35.

5. Lucatelli $P$, Argiro R, Bascetta S, et al. Single injection dual phase $\mathrm{CBCT}$ technique ameliorates results of trans-arterial chemoembolization for hepatocellular cancer. Transl Gastroenterol Hepatol. $2017 ; 2: 83$ 